\title{
GULF OF MEXICO \\ ORCA BASIN
}

\section{EN32-PC6}

The study of this core was undertaken in cooperation with James Kennett. The purpose was to establish the chronology of the Mississippi River melt water record (see Figs 7, 8; Table 6).

\section{REFERENCES}

Broecker, W S, Andrée, M, Wolfli, W, Oeschger, H, Bonani, G, Kennett, J and Peteet, D, in press, The chronology of the last deglaciation: Implications to the cause of the Younger Dryas event: Paleoceanography.

Kennett, J P, Elmstrom, K and Penrose, N, 1985, The last deglaciation in Orca Basin, Gulf of Mexico: High-resolution planktonic foraminiferal changes: Paleogeog, Paleoclimatol, Paleoecol, v 50, p 189-216.

Leventer, A, Williams, D F and Kennett, J P, 1982, Dynamics of the Laurentide ice sheet during the last deglaciation: Evidence from the Gulf of Mexico: Earth \& Planetary Sci Letters, v 59, p $11-17$.

tion in the Orca Basin, Gulf of Mexico: Marine Geology, v 53, p 23-40.

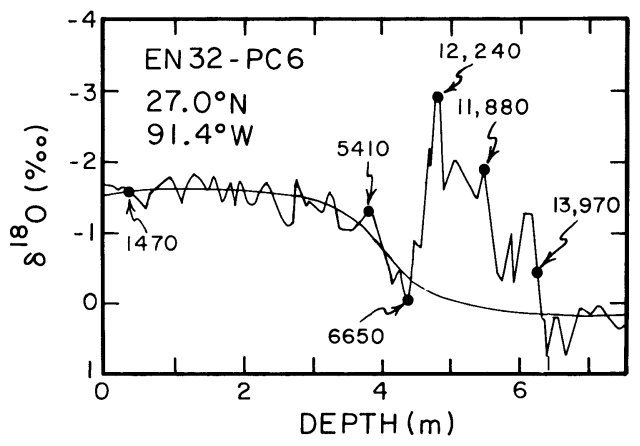

Fig 7. The ${ }^{18} \mathrm{O} /{ }^{16} \mathrm{O}$ record obtained on the shells of the planktonic species $G$ ruber from a Gulf of Mexico deep-sea core raised in the Orca Basin (Leventer, Williams \& Kennett, 1982). The smooth curve shows the record expected was the core from the open ocean. The large anomaly to more negative $\delta^{18} \mathrm{O}$ values is attributed to the discharge of glacial melt water from the Mississippi River. The ${ }^{14} \mathrm{C}$ analyses were carried out on hand-picked planktonic shells.

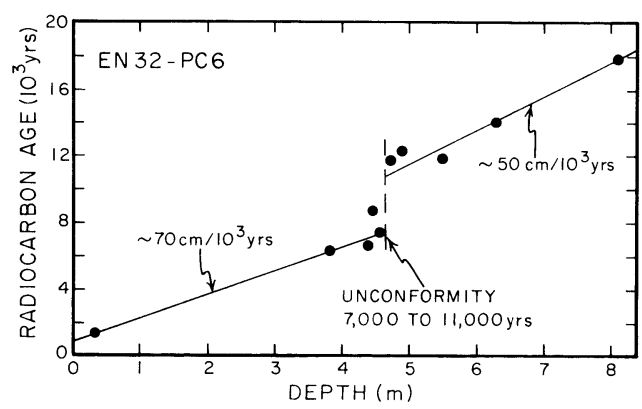

Fig $8 .{ }^{14} \mathrm{C}$ age $v s$ depth in Gulf of Mexico core EN32-PC6. The results suggest that a section of the record from ca $7000 \mathrm{BP}$ to ca $11,000 \mathrm{BP}$ is missing. The ${ }^{14} \mathrm{C}$ ages have not been corrected for the air-surface-sea age difference. 
TABLE 6

EN32-PC6 Gulf of Mexico Orca Basin Location $\left(26^{\circ} 57^{\prime} \mathrm{N}, 91^{\circ} 21^{\prime} \mathrm{W}\right)$ Depth $2280 \mathrm{~m}$

\begin{tabular}{|c|c|c|c|c|c|c|c|c|c|}
\hline $\begin{array}{l}\text { Depth } \\
(\mathrm{cm})\end{array}$ & $\begin{array}{c}\text { Coarse } \\
\text { fraction } \\
(\%)\end{array}$ & $\begin{array}{c}\text { Foram } \\
\text { sp }\end{array}$ & $\begin{array}{l}\text { Abund } \\
\text { (no./gm) }\end{array}$ & $\begin{array}{l}\text { Abund } \\
\text { (mgm/gm) }\end{array}$ & $\begin{array}{l}\text { No. tests } \\
\text { analyzed }\end{array}$ & $\begin{array}{l}\text { Weight } \\
\text { analyzed } \\
(\mathrm{mgm})\end{array}$ & $\begin{array}{c}\text { Date of AMS } \\
\text { analysis }\end{array}$ & $\begin{array}{l}\text { Age } \\
\text { (yr) }\end{array}$ & $\operatorname{Ref} *$ \\
\hline $23-29$ & - & M plank** & - & - & - & 10.2 & Mar 87 & $1470 \pm 120$ & 12 \\
\hline $380-382$ & - & M plank & - & - & - & 14.5 & Mar 87 & $5410 \pm 130$ & 12 \\
\hline $437-438$ & - & M plank & - & - & - & - & Sept 85 & $6650 \pm 110$ & 12 \\
\hline $442-443$ & 0.41 & M plank & - & - & - & - & Oct 86 & $8780 \pm 180$ & 12 \\
\hline $456-458$ & - & M plank & - & - & - & 7.2 & Mar 87 & $7360 \pm 160$ & 12 \\
\hline $470-472$ & - & M plank & - & - & - & 13.4 & Mar 87 & $11,690 \pm 210$ & 12 \\
\hline $485-487$ & - & $\underline{G}$ ruber & - & - & - & - & May 85 & $12,240 \pm 150$ & 12 \\
\hline $547-548$ & - & $\underline{G}$ ruber & - & - & - & 8.0 & Jan 87 & $11,880 \pm 210$ & 12 \\
\hline $627-629$ & - & M plank & - & - & - & - & Aug 86 & $13,970 \pm 410$ & 12 \\
\hline $808-810$ & - & M plank & - & - & - & 7.7 & Mar 87 & $17,860 \pm 370$ & 12 \\
\hline
\end{tabular}

* Publication no. in which radiocarbon date has been published (see Refereces cited) $* * \underline{M} \underline{\text { plank }}=$ mixed planktonic species

\section{ARCTIC OCEAN}

\section{FL-124}

This study was undertaken in cooperation with David Clark of the University of Wisconsin in order to confirm the previous estimates of low sedimentation rates in the Arctic Basin (see Table 7).

\section{REFERENCES}

Clark, D L, Andrée, M, Broecker, W S, Mix, A, Bonani, G, Hofmann, H J, Morenzoni, E, Nessi, M, Suter, M and Wolfli, W, 1986, Arctic Ocean chronology confirmed by accelerator ${ }^{14} \mathrm{C}$ dating: Geophysical Research Letters, v 13, no. 4, p 319-321.

TABLE 7

FL-124 Arctic Ocean Location $\left(78^{\circ} 14^{\prime} \mathrm{N}, 174^{\circ} 42^{\prime} \mathrm{W}\right)$ Depth $1517 \mathrm{~m}$

\begin{tabular}{|c|c|c|c|c|c|c|c|c|c|}
\hline $\begin{array}{l}\text { Depth } \\
(\mathrm{cm})\end{array}$ & $\begin{array}{c}\text { Coarse } \\
\text { fraction } \\
(\%)\end{array}$ & $\begin{array}{c}\text { Foram } \\
\text { sp }\end{array}$ & $\begin{array}{l}\text { Abund } \\
\text { (no./gm) }\end{array}$ & $\begin{array}{l}\text { Abund } \\
\text { (mgm/gm) }\end{array}$ & $\begin{array}{l}\text { No. tests } \\
\text { analyzed }\end{array}$ & $\begin{array}{c}\text { Weight } \\
\text { analyzed } \\
(\mathrm{mgm})\end{array}$ & $\begin{array}{l}\text { Date of } \\
\text { analysi }\end{array}$ & $\begin{array}{l}\text { AMS Age } \\
\text { s (years) }\end{array}$ & $\operatorname{Ref} *$ \\
\hline $0-1$ & - & $\underline{N} \operatorname{pach}(s)$ & - & - & - & - & May 85 & $9130 \pm 120$ & 8 \\
\hline $2-3$ & - & $"$ & - & - & - & - & $"$ & $15310 \pm 210$ & 8 \\
\hline $4-5$ & - & $"$ & - & - & - & - & $"$ & $31720 \pm 1280$ & 8 \\
\hline $8-9$ & - & $"$ & - & - & - & - & $"$ & $>41100$ & 8 \\
\hline
\end{tabular}

* Publication no. in which radiocarbon date has been published (see References cited) 